\title{
Tracking the Structural and Chemical Evolution of Nanostructured Materials by In-Situ Experiments
}

\author{
Zaoli Zhang $^{1,2}$, Jinming Guo ${ }^{1}$ and Reinhard Pippan ${ }^{1}$ \\ ${ }^{1 .}$ Erich Schmid Institute, Austrian Academy of Sciences, Leoben, Austria. \\ 2. Department of Materials Physical, Montanuniversität Leoben, Leoben, Austria.
}

Bulk nanostructured materials show extremely high strength compared to bulk materials, and have received unprecedented attention in material research also due to its potential applications in industry. Severe plastic deformation is an emerging and quite efficient route to generate nanocrystalline materials out of normally immiscible systems. However, the main issue on nanostructured materials is its thermal stability [1]. In this contribution, the thermal stability of nanostructured materials will be explored at a very high-spatial resolution using modern spherical transmission electron microscopy (TEM) via simultaneous in-situ imaging and spectroscopy analysis. $\mathrm{CuCr}$ nanostructured alloy was utilized for in-situ monitoring the structural evolution and chemical composition changes simultaneously.

A coarse-grained $\mathrm{Cu}-\mathrm{Cr}$ composite material $(43 \mathrm{wt} \% \mathrm{Cr}, 57 \mathrm{wt} \% \mathrm{Cu}$, and corresponding to 48 at $\%$ $\mathrm{Cr}$ and $52 \mathrm{at} \% \mathrm{Cu}$, respectively) was deformed by high pressure torsion (HPT) at room temperature. The initial microstructure of the composite consists of a $\mathrm{Cu}$ matrix with $\mathrm{Cr}$ particles (volume fraction of about $50 \%$, mean diameter of about $50 \mu \mathrm{m}$ ) produced by PLANSEE (Reutte, Austria). Disks with a diameter of $\sim 8 \mathrm{~mm}$ and a thickness $(t)$ of $\sim 0.8 \mathrm{~mm}$ were HPT deformed for different numbers of turns $n$ under a constant pressure of $6.25 \mathrm{GPa}$ with a rotation speed of 0.2 rotations/minute. HPT disks which were deformed for 25 turns (equal strains $=400$ ) were used for in-situ annealing in TEM at $25^{\circ} \mathrm{C}, 212^{\circ} \mathrm{C}$ and $414^{\circ} \mathrm{C}$ after preparing TEM specimens from the edge of the disk, where the highest strain has occurred.

For a comparison, normally immiscible $\mathrm{Cu}-\mathrm{Fe}$ alloy system was mechanically alloyed directly from blended powders and vacuum arc-melted bulk respectively which contain different levels of content of oxygen impurity, with a series of compositions $(100-x)$ at. $\% \mathrm{Cu}-x$ at. $\% \mathrm{Fe}(x=0,5,15,25,35)$, by means of high pressure torsion.

A JEOL $2100 \mathrm{~F}$ field emission microscope $(200 \mathrm{kV})$ equipped with an image-side $\mathrm{C}_{\mathrm{S}}$-corrector which possesses a $1.2 \AA$ resolutions at $200 \mathrm{kV}$ was used. The aberration coefficients were set to be sufficient small. The HRTEM images were taken under slightly over-focus.

The evolution of the structural and chemical composition in the nanostructured materials with temperature was tracked in real-time. It demonstrates that the nanostructured materials are not only subjected to a structural change but also to obvious chemical composition fluctuations upon annealing. The destabilization process in the nanostructured materials starts at a quite low temperature (Figure 1). Real-time imaging and composition determination reveal the concentration changes with temperature, and allows further analyzing the dynamic behavior in nanocrystalline materials in details, i.e. deducing the instantaneous diffusion coefficients and excess vacancy concentration generated by severely deformation, and interface sharpening with annealing is observed. The experiments demonstrated a direct approach to measure the excess vacancy concentration via measuring elemental profiles, except visualizing the morphology evolution. Essentially, the study uncovers the interplay between the thermal stability and chemical decomposition process of bulk nanostructured materials in real-time.[2]

On the other hand, the dynamic process in nanocrystalline materials is modified when the impurity level (i.e. oxygen) in nanostructured materials is higher. One example, such as $\mathrm{Cu}-\mathrm{Fe}(25 \%$ wt $\mathrm{Fe})$ deformed using powders precursors which contain a different content of oxygen, is given, demon- 
strating a total different behavior when annealed. Surprisingly, nano-oxides form firstly (Figure 2), and then followed by a decomposition of supersaturated solid solution upon heating $[3,4]$.

\section{References:}

[1] RZ Valiev et al, Mater Res Lett 4 (2016), 1.

[2] Zaoli Zhang et al, Acta Materialia 138 (2017), 42.

[3] Jinming Guo et al, Nature communications 9 (2018), 946.

[4] The authors acknowledge funding from the by the Austrian Science Fund (FWF): No. P27034 N20. Prof. Gerhard Dehm, Max-Planck-Institut für Eisenforschung, D-40237 Düsseldorf, Germany, is thanked for his useful discussions.
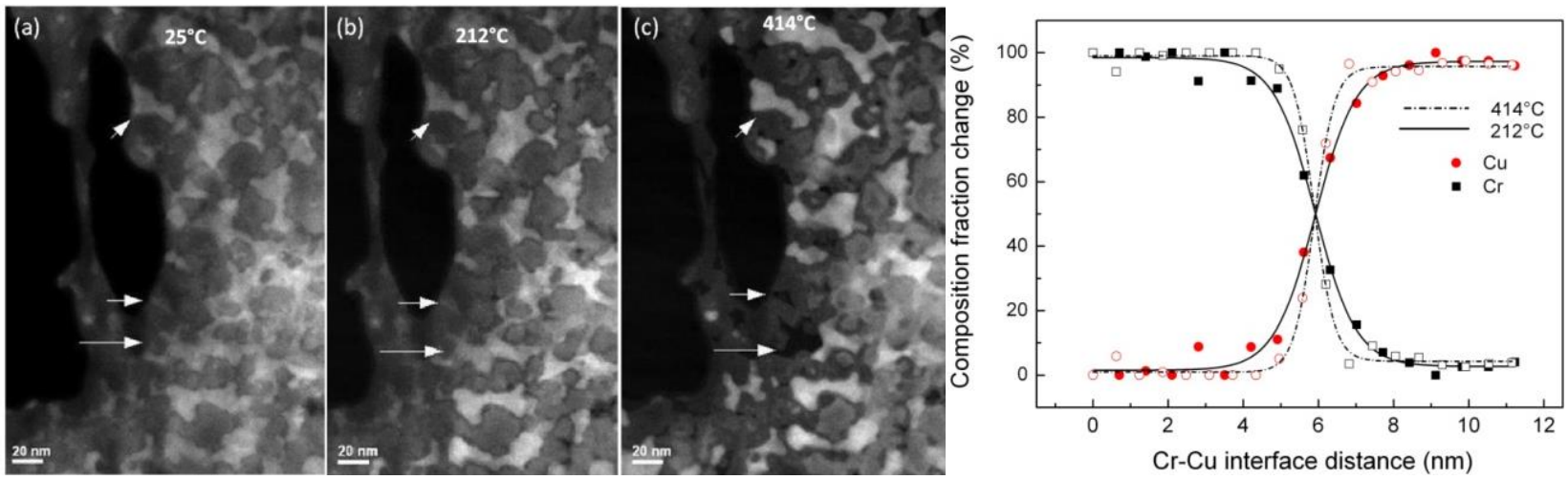

Figure 1. Left (a) (b) and (c): HAADF STEM image demonstrating the nanocrystalline structural evolution process; (a) $25^{\circ} \mathrm{C}$, (b) $212^{\circ} \mathrm{C}$, (c) $414{ }^{\circ} \mathrm{C}$. Particular locations labeled by arrows. Some pores are formed immediately upon $414 \mathrm{C}$. Right panel: Elemental profile crossing one $\mathrm{CrCu}$ boundary.
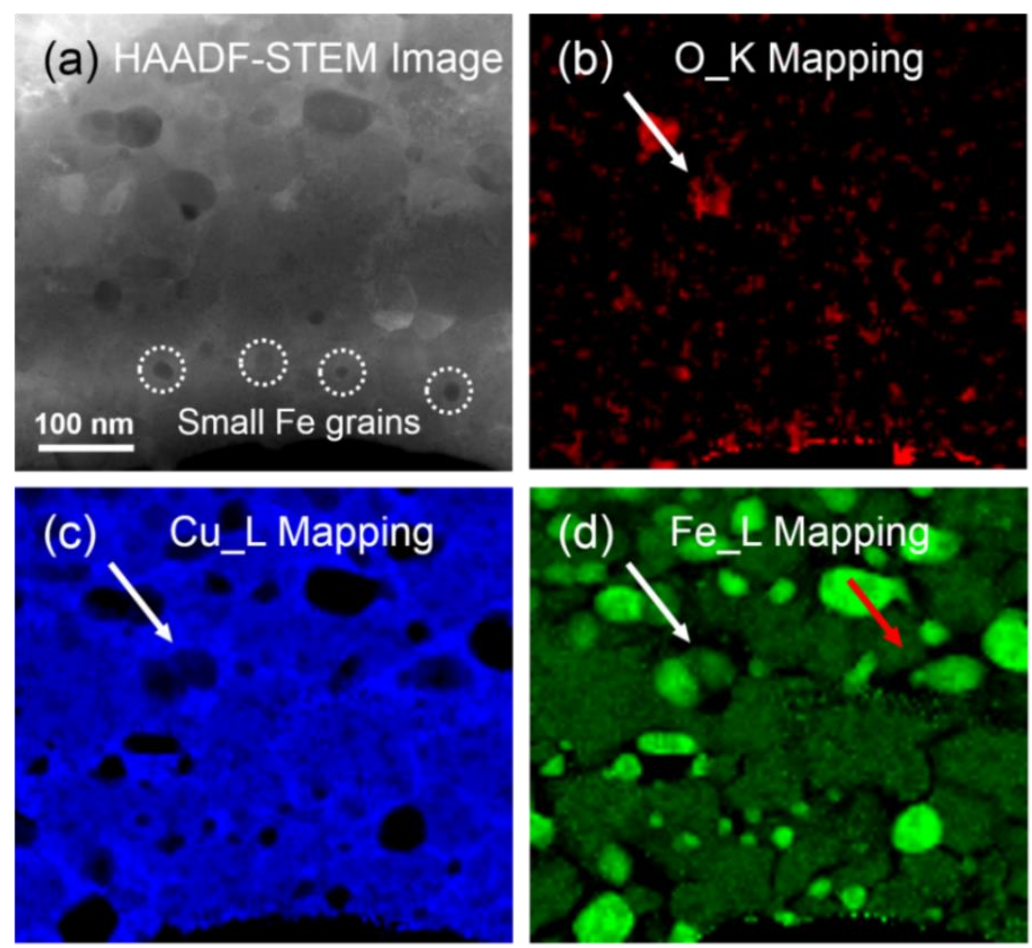

Figure 2. EELS elemental mapping of annealed sample (CuFe nanostructured materials) at $420^{\circ} \mathrm{C}$. (a) HAADF-STEM image. (b) O_K mapping. (c) Cu_L mapping. (d) Fe_L mapping. 